\title{
A Laser Scribed Graphene Oxide and Polyimide Hybrid Strain Sensor
}

\author{
SHAYAN Naveed ${ }^{a}$, TAYYABA Malik, MUHAMMAD Munir, \\ and MOHAMMAD Ali Mohammad ${ }^{\mathrm{b}, *}$
}

\author{
School of Chemical and Materials Engineering (SCME), National University of Sciences and \\ Technology (NUST), Sector H-12, Islamabad 44000, Pakistan \\ ashayan_nse3@scme.nust.edu.pk, bdr.ali@scme.nust.edu.pk
}

Keywords: Laser scribing, LIG, polyimide, rGO, strain sensor, gauge factor

\begin{abstract}
Strain sensors are devices used in applications such as electronic skin, prosthetic limbs, e-textiles, and many more, for measuring the physical elongation of a desired structure under a given or applied force. An artificial throat, using a strain sensor, was recently developed as an aid for speech impaired individuals. Strain sensors have been developed using graphene and polydimethylsiloxane (PDMS), with a reported gauge factor ranging from 5 120. In this work, a strain sensor was developed through laser scribing. Laser scribing is a recent and facile technology used for printed electronics, whereby complex geometries and patterns can be drawn very easily. The laser scribing method relies on the property of certain materials to form a graphene-like conductive material upon irradiation by lasers. Polyimide and graphene oxide (GO) are two such materials. In these experiments, $2 \times 3 \mathrm{~cm}$ sheets of polyimide were taken and a $1 \times 1 \mathrm{~cm}$ box on the sheet was printed using a laser patterning setup of $450 \mathrm{~nm}$ wavelength. Graphene oxide solution was drop-casted on the reduced polyimide sheet of $1 \times 1 \mathrm{~cm}$, to increase its sensitivity, and then the drop-casted graphene oxide is reduced using the same laser. The strain sensor was characterized by a micro-strain testing machine. In the preliminary results, the normalized resistance was plotted against strain and the gauge factor (GF) was calculated. The intensity of the laser was varied, and different gauge factors were calculated. The effect of laser intensity was investigated. The gauge factor was found to be in the range of 49-54 and was compared with the polyimide reduced strain sensor (without drop-casting process).
\end{abstract}

\section{Introduction}

In recent years, strain sensors have caught a lot of attention as they have numerous applications in different industries such as health, aerospace, automotive and electronic communications. Strain sensors are devices which exhibit a change in the electrical characteristics upon a mechanical strain. A good strain sensor must have a high sensitivity or in other words a high GF, durability, repeatability, low response time, easy operation and a low cost of fabrication. There are various applications for these devices such as structural health monitoring [1], heart rate monitors, electronic skins [2], motion capture devices [3,4] and many more. Conventional strain gauges use a metallic foil pattern to sense the strain, however, these are not very sensitive or efficient. The GF for such a device is low i.e., $\sim 2-5$.

In recent history, smart carbon-based materials are being employed such as graphene [5] and CNTs [6] due to their higher GF. These materials exhibit piezo-resistivity (change in resistance due to deformation). Different types of methods have been adopted to fabricate these devices. Using Chemical Vapour Deposition (CVD) Zhao et.al were able to achieve a GF over 300 [7]. ZnO Nanowires are also used with extremely high GFs 300-1250 [8,9]. Semiconducting SWCNTs also showed a very high GF of 600-1000 [10] whereas a metallic Ag nanowires/elastomer nanocomposite was synthesized which had a GF of 4-16.6 [11]. Different graphene composites were made by incorporating graphene in a polymer or elastomer matrix such as polydimethylsiloxane (PDMS) with GFs in the range of 34.3 - 48.9 [12].

Most of the methods mentioned above are either very expensive or time consuming. In this study, a flexible polyimide substrate is used which undergoes photo-thermal reduction upon being 
subjected to a laser. A porous structure of carbon is formed which is similar to graphene. This material is referred to as laser scribed graphene (LSG). Reduction of graphene oxide by laser scribing was introduced by El-Kady et al. by which they synthesized electrochemical capacitors and micro-capacitors [13] by using a DVD burner with LightScribe software. The concept of irradiating polymers to increase their conductivity was given by Schumann in 1991[14]. In these experiments, polyimide was irradiated increasing its conductivity by 15 orders of magnitude. The same concept was used by Lin.et al, in which a $\mathrm{CO}_{2}$ laser was used to irradiate polyimide and the authors named it Laser Induced Graphene (LIG). Interdigitated patterns on polyimide were printed to form microsuper-capacitors [15]. Since then, different devices have already been made such as artificial throat, pressure sensor and a strain sensor with a GF up to 9.49 [16-18].

In this study, we aim to make a hybrid structure on a flexible substrate. A polyimide sheet is reduced with a laser. The laser has an X-Y stage which is controlled by software. In this software, we can import pictures according to our specifications. Subsequently, GO solution is drop-casted on the reduced polyimide area. The GO solution is then reduced by a low intensity laser burst. This will form a hybrid structure of LIG and rGO which has a high conductivity and high GF compared to conventional strain gauges or conventional graphene composites. This strain sensor only takes 45 minutes to fabricate, hence it can be produced on a large scale very easily. There is no chemical waste in the fabrication process, so it is also environmentally friendly.

\section{Experimentation}

Materials Required. The materials required for experiments are $0.25 \mathrm{~mm}$ thick sheet of polyimide, GO solution, quick drying silver paste (Agar technologies), high temperature KAPTON tape and Copper tape.

Instrumentation. Instruments required for sample preparation and characterization are OSRAM diode laser (450nm wavelength) for Laser scribing of Polyimide and Graphene Oxide, STOE thetatheta Diffractometer (XRD) for XRD analysis of the prepared samples, JEOL Analytical SEM (JSM-6490A) for surface morphology of LIG and rGO/LIG, Biologic EC-Lab (VSP) for electrical measurements and Micro-strain test rig for strain sensing.

Fabrication of Strain Sensor. Firstly, graphene oxide is synthesized by a modified Hummer's process [19]. A $3.7 \mathrm{mg} \mathrm{mL}^{-1}$ solution [20] is made by dispersing $74 \mathrm{mg}$ GO powder in $20 \mathrm{~mL}$ distilled water. The solution is sonicated for 2 hours. Polyimide sheet is cut into $2 \times 3 \mathrm{~cm}$ rectangles and is subjected to laser induced reduction as shown in Fig. 1. The laser power is varied for different samples. The desired shape is fed into the laser software $(1 \times 1 \mathrm{~cm}$ square in our case). After the polyimide is reduced, $0.5 \mathrm{~mL}$ sonicated GO dispersion is drop casted on the exposed square.

Fig.1. The process flow for the fabrication of $\mathrm{LIG} / \mathrm{rGO}$ strain sensor. a) $2 \times 3 \mathrm{~cm}$ polyimide sheet, b) LIG formed, c) GO solution is drop-casted, d) the sample is rotated and exposed to laser again, e) quick drying silver paste is applied to form electrodes, and f) copper tape is attached on the electrodes.

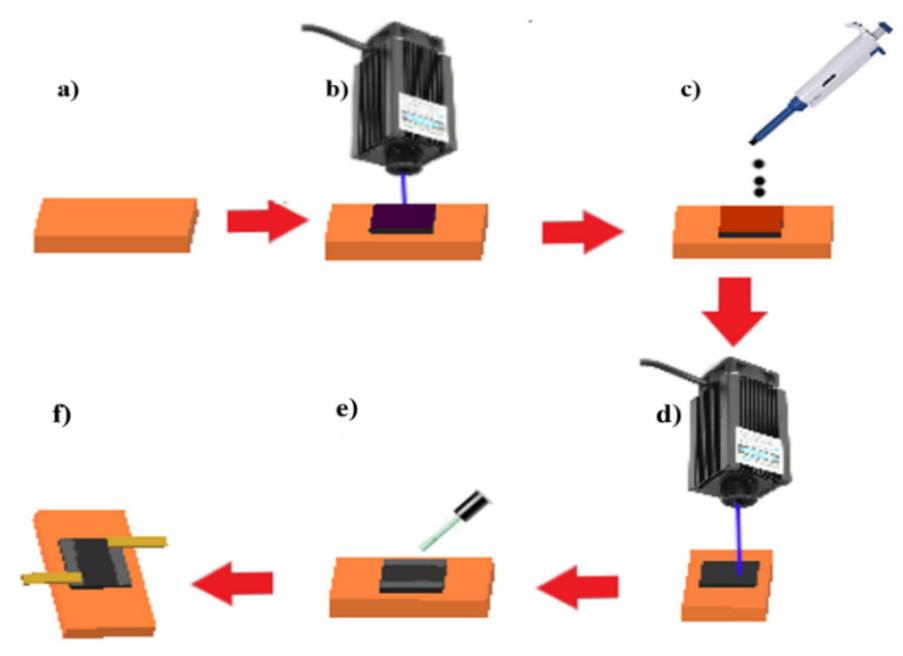


Samples are left to dry in an oven. This time the sample is rotated at $90^{\circ}$ and is reduced once again by the laser to form the hybrid structure of LIG and rGO. The second laser reduction is done at $52 \mathrm{~mW}$ power for all samples as shown in Fig.1. After the sample is made, electrodes are formed by quick drying silver paste and the copper tape is attached to the electrodes for different types of measurements such as I-V characteristics and GF measurement.

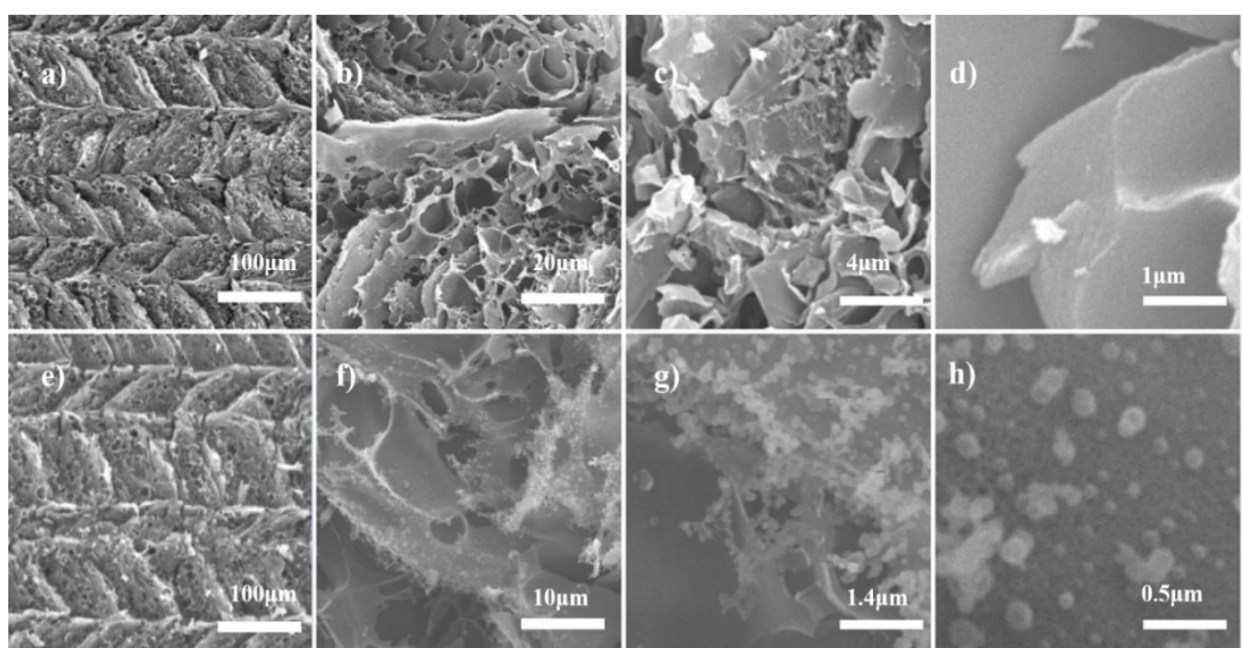

Fig. 2. SEM results for both LIG and LIG/rGO. All images are taken from the sample exposed to $170 \mathrm{~mW}$. The top view SEM images a-d and e-h are for LIG and LIG/rGO, respectively.
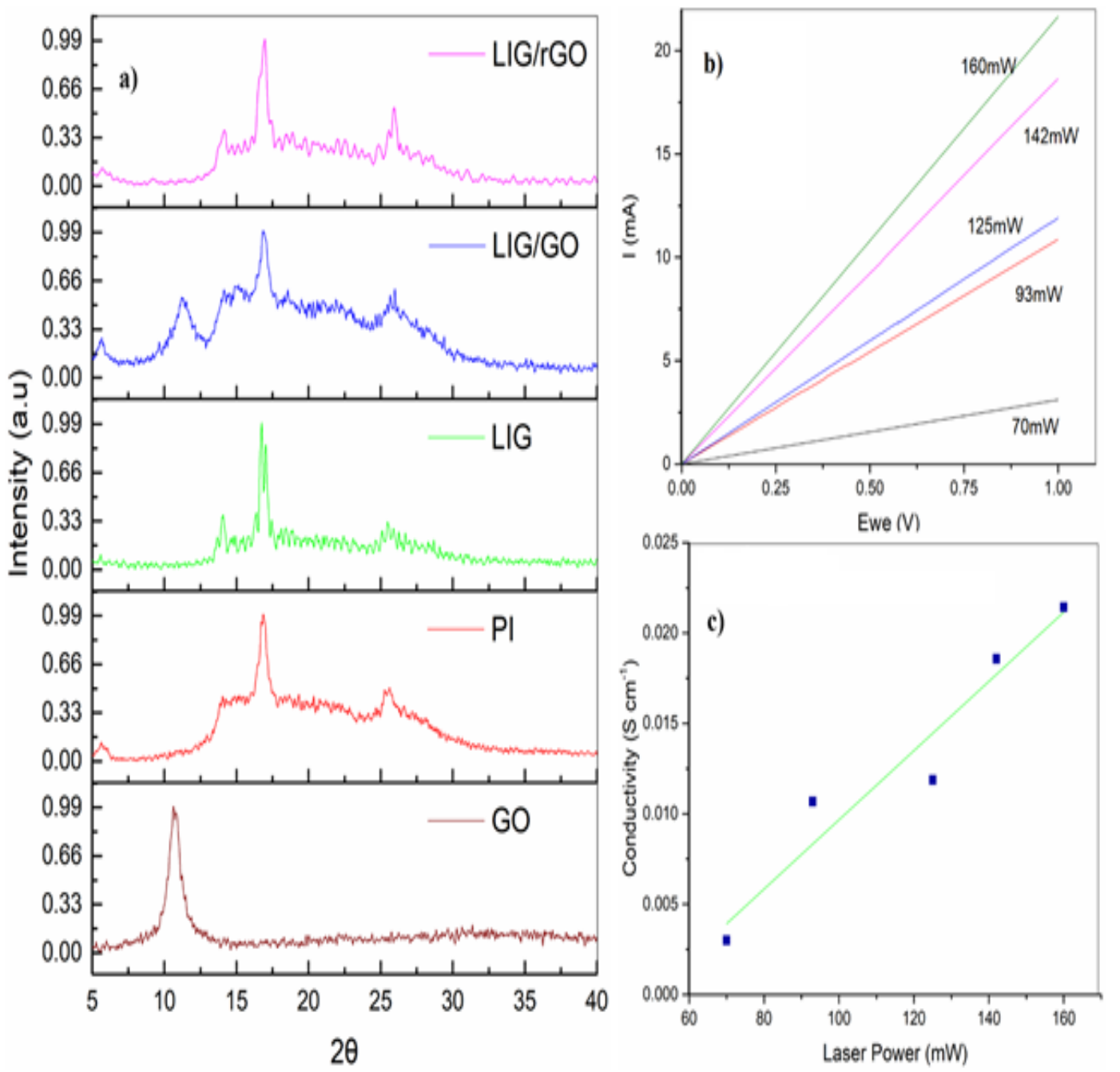

Fig. 3. a) XRD performed at different stages of the fabrication process. b) I-V characteristics of 5 different LIG/rGO samples with increasing laser power, c) Conductivity vs laser power plot and their relation. 
X-ray diffraction (XRD) for GO powder and polyimide sheet was performed at the start. Afterwards, the XRD was performed at different stages of the fabrication process as illustrated in Fig 3a. GO powder shows a peak at $10.9^{\circ}$ with a $d$-value of $8.24 \AA$ [21] confirming the species as graphene oxide. As the polyimide is not crystalline, no sharp peaks were formed. After the first laser exposure, the peaks started to become sharper because a more crystalline structure is being formed out of a completely amorphous polymer. As the GO solution is drop-casted, a peak at $12^{\circ}$ is observed. After the second exposure to the laser, the $12^{\circ}$ peak disappears suggesting that the GO has been converted to rGO. This is also confirmed as the peak at $26^{\circ}$ becomes more pronounced.

Device Characterization. Samples at different laser power were fabricated and characterized using the Biologic EC-Lab. Using this equipment, I-V characteristics of these samples were calculated.
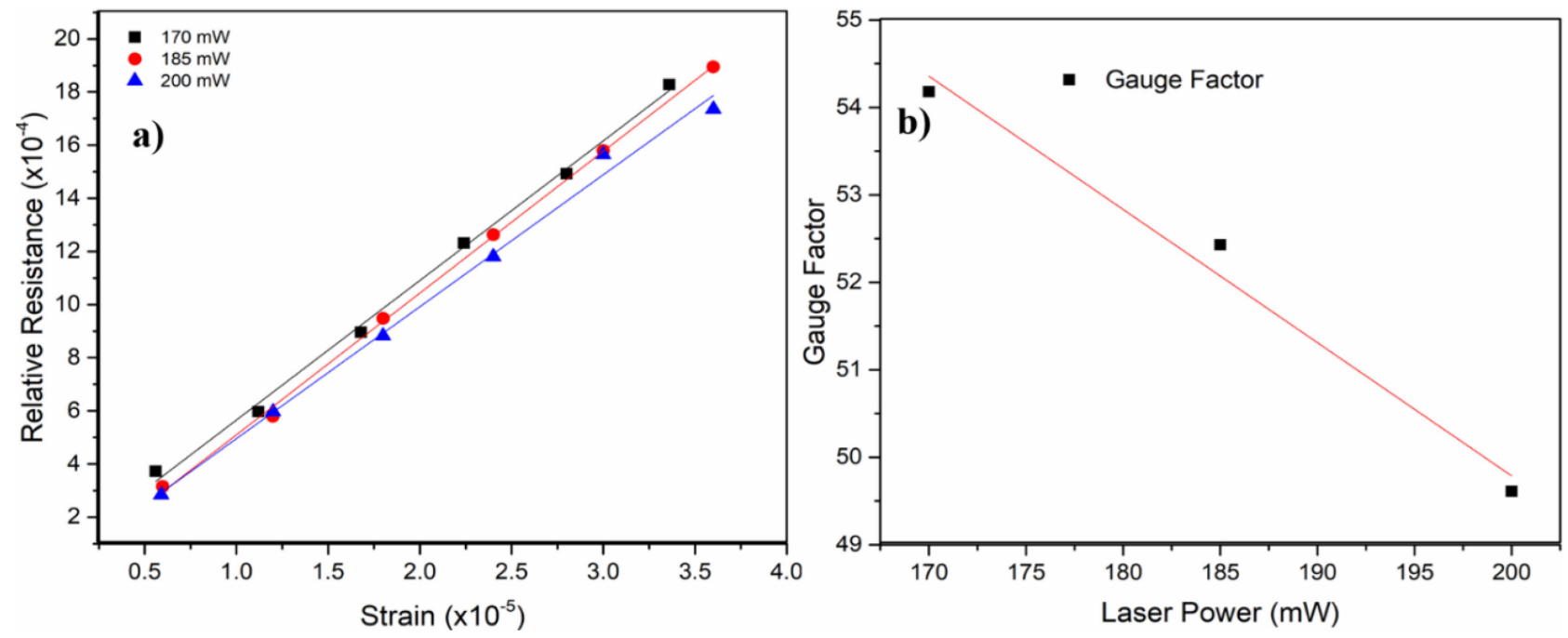

Fig. 4. Strain sensing plots a) Relative resistance vs strain for three different laser powers, and the b) relationship between laser power and GF.

The slopes of the I-V characteristics increase as shown in Fig.3b with increasing laser power. This indicates that the photo-thermal reduction process gets more pronounced, and the stacking or overlapping between the graphene layers increases with the increase in laser power. The conductivities were also calculated by analysing these graphs and Fig.3c is generated which shows that conductivity increases with the increase in laser power. The highest conductivity was at 160 $\mathrm{mW}$ which was $0.214 \mathrm{~S} \mathrm{~cm}^{-1}$ and the lowest was at $70 \mathrm{~mW}$ which was $0.003 \mathrm{~S} \mathrm{~cm}^{-1}$. It is to be noted that the linear behaviour is for this range only and entirely different behaviour could be expected in a range consisting of much higher laser powers [22].

The strain sensing was performed on a micro-strain test rig. This rig was made up of a $150 \mathrm{~mm}$ triangular cantilever attached to a depth micro-meter which controls the strain. The samples were pasted on the cantilever and connected to a digital multi-meter. The strain on the cantilever was produced by the depth micro-meter and the resistance was noted down. For quality purposes, the GF of the strain sensor was calculated. The gauge ensures whether the sensor is highly sensitive towards strain or not. In order to calculate the GF, relative resistance vs strain is plotted, as the formula for gauge factor is given below;

$$
G F=(\Delta R / R) / \varepsilon
$$

where $\Delta R / R$ being the relative change in resistance and $\varepsilon$ is the strain.

Hence the slope of this plot would provide the GF of the sample shown in Fig 4a. Therefore, the GFs for these samples are 54.18 at $170 \mathrm{~mW}, 52.43$ at $185 \mathrm{~mW}$ and 49.61 at $200 \mathrm{~mW}$. As Fig 4.b illustrates, the GFs are decreasing with increasing laser power. 


\section{Discussion}

Although reduced graphene oxide has been investigated for several years, still there are numerous mechanisms which are unknown or unclear. When discussing the microstructure of laser induced graphene and its relation with electrical conductivity, firstly, there is a direct relation between the thickness of the LIG film and the resistance of the device. As the thickness is increased the resistance decreases. Further, the thickness can be correlated with the laser power, as the laser power increases the thickness of the LIG film also increases. Therefore, with increasing laser power, the resistance decreases. This decrease in resistance is due to overlapping of graphene sheets both in rGO and LIG as they are pinned down while being treated with the laser.

Fig. $4 \mathrm{a}$ depicts that the relative resistance increases with increasing strain. Further, the GF decreases with increasing laser power as shown in Fig. 4b. There are many theories which are proposed, and a relatively facile explanation is that the conductivity depends on the contact resistance and overlapping area between the adjacent graphene sheets and so does the strain response. As the reduced area undergoes tensile stress, the individual graphene sheets are pulled apart hence hindering the current flow and the resistance is increased. On the other hand, when compressive stresses are applied, the individual graphene sheets overlap each other and the resistance decreases. The GF increases with an increase in resistance, or in other words, the GF increases when the flake density or over-lap area is low. Every graphene flake acts as a sub-circuit, in a stretched state some of the connections between these sub-circuits are already broken due to which the change in resistance is faster. The same reasoning applies for samples with low density graphene flakes and samples made with low power. They would have a lower number of sub circuits and in a stretched state a faster resistance change will be exhibited.

In an un-stretched state, the graphene sheets are closer to each other and the overlapping area is large; however, under small strain the graphene sheets are pulled apart and the overlapping area decreases. This behaviour (decrease of overlapping area) continues as the strain is increased [23].

\section{Conclusion}

A clear relation between laser power, conductivity, resistance and GF has been developed. The GF is very high and can be used in motion capture applications or even in bio-sensors. All of the GF calculations showed a very linear relation, hence this strain sensor is reliable. A laser power of $170 \mathrm{~mW}$ showed the most accurate result with the highest GF of 54.2.

\section{Acknowledgements}

Dr. Rizwan Hussain from the National University of Sciences and Technology (NUST) is acknowledged for (a) useful technical discussions, and (b) partial support via Higher Education Commission (HEC) of Pakistan grant number 6040/Federal/NRPU/R\&D/HEC/2016. Prof. Aslam Baig and their staff at the National Center for Physics (NCP) are acknowledged for measurement of the laser system parameters. The authors are grateful to Shazrah Shahzad, Ramsha Khan and Adeel Riaz of the School of Chemical and Materials Engineering (SCME), NUST, for their useful discussions. Special mentions for Lab Tech. Shams Ud Din and Lab Tech. Khurram Shahzad (SCME, NUST) for assistance in obtaining SEM and XRD data.

\section{References}

[1] V. Eswaraiah, K. Balasubramaniam, and S. Ramaprabhu, Functionalized graphene reinforced thermoplastic nanocomposites as strain sensors in structural health monitoring. J. Mater. Chem., 21 (2011) 34 .

[2] Z. Lou, S. Chen, L. Wang, K. Jiang, and G. Shen, An ultra-sensitive and rapid response speed graphene pressure sensor for electronic skin and health monitoring. Nano Ener., 23 (2016) 7-14.

[3] C. S. Boland, U. Khan, C. Backes, A.O’Neill, J. McCauley, et al., Sensitive, High-Strain, HighRate Bodily Motion Sensors Based on Graphene-Rubber Composites. ACS Nano, 8 (2014) 88198830. 
[4] C.Yan, J. Wang, W. Kang, M. Cui, X. Wang, et al. (2014). Highly Stretchable Piezoresistive Graphene-Nanocellulose Nanopaper for Strain Sensors. Adv. Mater., 26 (2014) 2022.

[5] S. H. Bae, Y. Lee, B. K. Sharma, H. J. Lee, J. H. Kim, et al., Graphene-based transparent strain sensor. Carbon, 51(2013) 236-242.

[6] I. Kang, M. J. Schulz, J. H. Kim, V. Shanov, and D. Shi, A carbon nanotube strain sensor for structural health monitoring. Smart Mater. Struct., 15 (2006) 737.

[7] J. Zhao, C. He, R. Yang, Z. Shi, M. Cheng, et al., Ultra-sensitive strain sensors based on piezoresistive nanographene films. Appl. Phy. Lett., 101 (2012) 63112.

[8] X. Xiao, L. Yuan, J. Zhong, T. Ding, Y. Liu, et al. (2011). High-Strain Sensors Based on ZnO Nanowire/Polystyrene Hybridized Flexible Films. Adv. Mater., 23 (2011) 5440.

[9] J. Zhou, Y. Gu, P. Fei, W. Mai, Y. Gao, et al., Flexible Piezotronic Strain Sensor. Nano Lett., 8 (2008) 3035-3040.

[10] J. Cao, Q. Wang and H. Dai. Electromechanical Properties of Metallic, Quasimetallic, and Semiconducting Carbon Nanotubes under Stretching. Phys. Rev. Lett., 90 (2003) 157601.

[11] M. Amjadi, A. Pichitpajongkit, S. Lee, S. Ryu and I. Park. Highly Stretchable and Sensitive Strain Sensor Based on Silver-Elastomer Nanocomposite. ACS Nano, 8(2014), 5154-5163.

[12] A. Nakamura, T. Hamanishi, S. Kawakami and M. Takeda. A piezo-resistive graphene strain sensor with a hollow cylindrical geometry. Mater. Sci. Engineer. B: Solid-State Materials for Adv. Technol., 219 (2017) 20-27.

[13] M. F. El-Kady, V. Strong, S. Dubin and R. B. Kaner. Laser Scribing of High-Performance and Flexible Graphene-Based Electrochemical Capacitors. Sci., 335 (2012) 1326-1330.

[14] M. Schumann, R. Sauerbrey and M.C. Smayling. Permanent increase of the electrical conductivity of polymers induced by ultraviolet laser radiation. Appl. Phy. Lett., 58 (1991) 428430.

[15] J. Lin, Z. Peng, Y. Liu, F. Ruiz-Zepeda, R. Ye, et al. Laser-induced porous graphene films from commercial polymers. Nat. Commun., 5 (2014) 1-8.

[16] L.-Q. Tao, H. Tian, Y. Liu, Z.-Y. Ju, Y. Pang, et al. An intelligent artificial throat with soundsensing ability based on laser induced graphene. Nat. Communicat., 8 (2017) 14579.

[17] H. Tian, Y. Shu, X.-F. Wang, M.A. Mohammad, Z. Bie, et al. A graphene-based resistive pressure sensor with record-high sensitivity in a wide pressure range. Scientif. Rep., 5 (2015) 8603.

[18] H. Tian, Y. Shu, Y.-L. Cui, W.-T. Mi, Y. Yang, et al. Scalable fabrication of high-performance and flexible graphene strain sensors. Nanoscale, 6 (2014) 699-705.

[19] D. C. Marcano, D. V Kosynkin, J. M. Berlin, A. Sinitskii, Z. Sun, et al. (2010). Improved Synthesis of Graphene Oxide. ACS Nano, 4 (2010) 4806-4814.

[20] M. Gamil, H. Nageh, I. Bkrey, S. Sayed, A. M. F. El-Bab, K. Nakamura, et al. Graphene-based strain gauge on a flexible substrate. Sens. Mater., 26 (2014) 699-709.

[21] Z. Fan, K. Wang, T. Wei, J. Yan, L. Song, and B. Shao, An environmentally friendly and efficient route for the reduction of graphene oxide by aluminum powder. Carbon, 48 (2010) 16861689.

[22] R. Kumar, R.K. Singh, D.P. Singh, E. Joanni, R.M. Yadav, and S.A. Moshkalev, (2017). Laser-assisted synthesis, reduction and micro-patterning of graphene: Rec. prog. Appli., Coordi. Chem. Rev. (Supplement C), 342 (2017) 34-79.

[23] D.Y. Wang, L.-Q. Tao, Y. Liu, T.-Y. Zhang, Y. Pang, et al. High performance flexible strain sensor based on self-locked overlapping graphene sheets. Nanoscale, 8 (2016) 20090-20095. 